\title{
How to navigate the myosin-V motor through the actin network
}

Peter Spieler*1, Angela Oberhofer*1, Dennis Zimmermann², Edwin Brames ${ }^{1}$, Alistair N. Hume ${ }^{3 \dagger}$, and Zeynep Ökten ${ }^{1 \dagger}$

${ }^{1}$ Physik Department E22, Technische Universität München, James-Franck-Strasse 1, D-85748 Garching, Germany.

2Massachusetts Institute of Technology, Koch Institute for Integrative Cancer Research, 77 Massachusetts Ave, Cambridge, MA 02139-4307, U.S.A.

${ }^{3}$ School of Life Sciences, University of Nottingham, Medical School, Queen's Medical Centre, Nottingham, NG7 2UH, UK.

*These authors contributed equally to this work.

${ }^{\dagger}$ Corresponding authors:

Alistair N. Hume

School of Life Sciences

University of Nottingham

Medical School

Queen's Medical Centre

Nottingham, NG7 2UH, UK.

Alistair.Hume@nottingham.ac.uk

+441158230102

Zeynep Ökten

Physik Department E22

Technische Universität München

James-Franck-Straße 1

D-85748 Garching, Germany

zoekten@ph.tum.de

+498928912885 


\section{Summary}

2 Myosin-V (MyoV) is a ubiquitous motor protein that transports an astonishingly diverse

3 set of cargos on the actin network in eukaryotes. Phosphorylation-dependent

4 processes often regulate MyoV-mediated cargo transport, molecular details of which

5 remain largely unknown. We previously showed that phosphorylation regulates MyoV's

6 switching from microtubules onto actin filaments, not its motor activity. Regulation of

7 switching at reconstituted microtubule-actin-crossings in fact sufficed to recapitulate the MyoVa-driven redistribution of pigment-organelles in amphibian melanophores. However, in those cells, MyoVa also encounter many actin-actin crossings. Here, we show that isolated MyoVa motors switch with equal probabilities at reconstituted actinactin-crossings. Under the control of its adaptor-protein melanophilin (Mlph), however, the motor differentiates between the actin filaments at crossing points in a phosphorylation-regulated manner. Whereas phosphorylation of Mlph forced about $\sim 2 / 3$ of MyoVa to ignore the intersections, dephosphorylation completely reversed this behavior and forced $\sim 2 / 3$ to switch. We show that the filament-binding domain (FBD) of Mlph controls this switching behavior. This property evolved in amphibians, but not in the early vertebrate zebrafish. By protein engineering, we demonstrate that changes of a few residues are sufficient to impart actin-binding capability onto the zebrafish Mlph. We thus unmask the molecular beginnings of dual filament binding in Mlph that allow it to control the switching behavior of MyoVa at cytoskeletal crossings. We therefore propose a direct link between intracellular phosphorylation activity and the adaptor-protein, not to regulate MyoVa activity, but to navigate the motor through the 
24 Key words: Myosin-V motor, actin network, PKA, transport regulation

\section{Significance statement}

26 In virtually all eukaryotic cells, numerous myosin motors have to navigate through an

27 elaborate actin network for timely transport of intracellular cargo. Here, we unmask an

28 unintuitive regulation of the myosin-Va motor that is involved in pigment organelle

29 transport. We demonstrate that myosin-Va differentiates between the same actin

30 filaments and displays regulated switching at reconstituted actin-actin crossings, an

31 unexpected behavior that has been predicted from previous theoretical work. We trace

32 this regulation back to the adaptor protein of the myosin-Va motor and show that this

33 regulation was present in amphibian but had not evolved in the early vertebrate

34 zebrafish. Notably, we demonstrate that the evolution of actin-binding capability is

35 achieved by changing a few residues in the adaptor protein. 


\section{Introduction}

How cells regulate kinesin, dynein, and myosin motors to ensure timely positioning of intracellular cargo at the right place is of outstanding interest. Instead of working alone, different motor types often team up on a given cargo. Particularly convoluted cases involve kinesin, dynein, and myosin motors simultaneously, all of which appear to know how to take turns to achieve a correct and timely positioning of cargo on the microtubule and actin cytoskeleton.

A well-known example of such convoluted transport occurs in melanophores from early and lower vertebrates to give rise to physiological color change. These specialized cells produce large, pigment-filled organelles, termed melanosomes, onto which they simultaneously recruit kinesin, dynein, and myosin motors (1-7). Fish and amphibians make use of the prototypical cAMP-PKA-dependent regulatory pathway to consciously control the deployment of these three different types of motors (Fig. 1A and B) (8-11). When external cues lead to an increase of intracellular PKA activity, melanosomes disperse throughout the cell on the actin network (Fig. 1A). Conversely, when PKA activity is decreased, and phosphatase activity increased, melanosomes switch from the actin onto the microtubule network to cluster in the cell center. This dynamic redistribution of large black granules in turn controls the light-scattering properties of the cell, allowing the organism to appear light or dark depending on external cues.

However, while fish and frog models make use of kinesin, dynein, and myosin for regulated melanosome redistribution, they differ substantially in the functional use of the microtubule and actin cytoskeletal systems to achieve voluntary color change. Fish and amphibians both depend on the dynein motor to cluster their melanosomes in the cell center upon decrease of PKA activity $(2,3)$. Dispersal of the organelles as a response to increased PKA activity, on the other hand, requires the presence of the MyoVa/actin system in the amphibian (5), but not in fish model (12). In the absence of 
A

Dispersion: increased light scattering
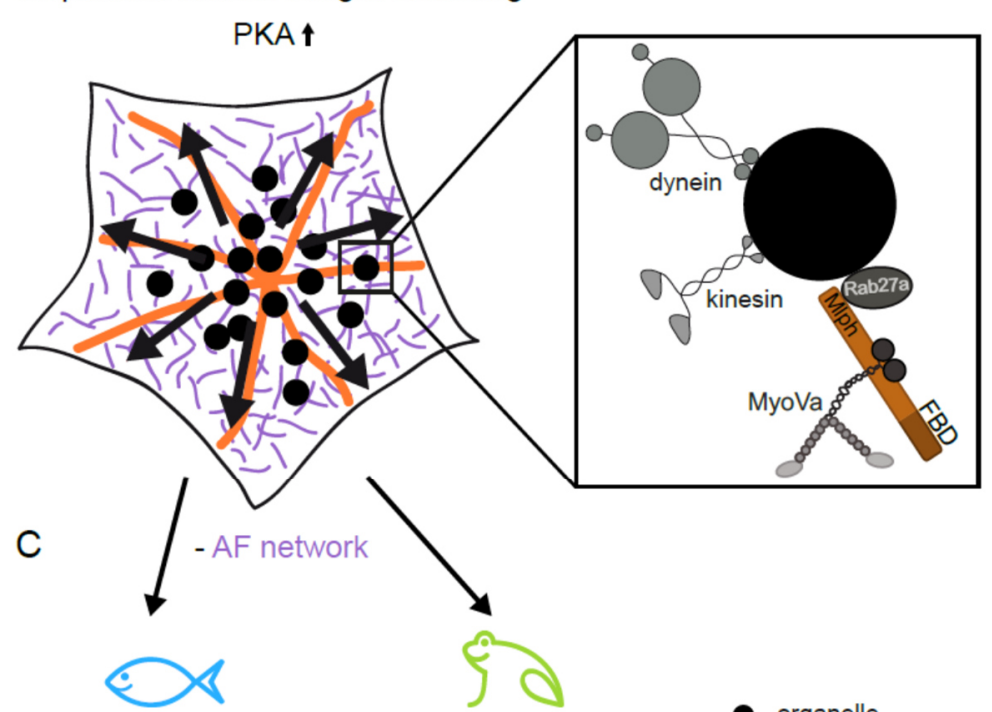

fish: hyperdispersion

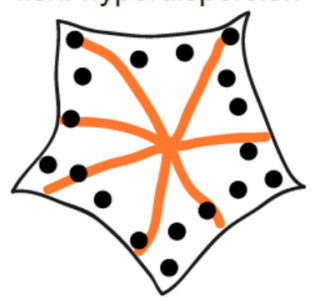

\section{B}

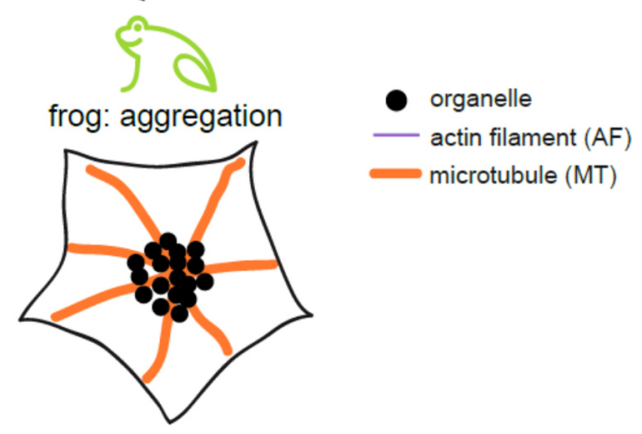

Fig. 1: Fish and amphibians differ in use of the microtubule and actin networks in melanosome transport. (A) Illustration of melanosome dispersion in melanophores. The cell increases the scattering of light by dispersing the large black melanosomes as a response to 
increased PKA activity (black arrows indicate the overall direction of transport). (B) Kinesin, dynein, and myosin motors are found simultaneously on the melanosome surface. Recruitment of MyoVa is achieved by the Rab27a GTPase and the melanophilin (Mlph) adaptor protein (FBD indicates the C-terminal filament-binding domain). (C, left panel) Removal of the actin network prompts hyperdispersion in fish, i.e. the kinesins motor transport the melanosomes to the end of the microtubules. (Right panel) Frog fails to display hyperdispersion and melanosomes are transported to the cell center by the dynein motors after removal of the actin network, demonstrating the essential role of the actin network system to disperse melanosomes in amphibians.

We previously provided a molecular rationale for these observations by studying the behavior of PKA-phosphorylated and dephosphorylated Rab27a/Mlph/MyoVa complexes from Xenopus frog and zebrafish at in vitro reconstituted microtubule-actin crossings (13). Consistent with the hypothesis that functional crosstalk between the microtubule and actin networks is required for the cell-wide redistribution of melanosomes in the frog model, the amphibian XIRab27a/XtMlph/XIMyoVa complex displayed regulated switching at reconstituted microtubule-actin crossings in vitro, but not the homologous complex from fish (13). We eventually pinpointed this regulation to the FBD of the XtMlph adaptor protein in the amphibian transport complex (13). While the phosphorylation-state of XtMlph dictated the switching behavior of its XIMyoVa at microtubule-actin crossings, up-regulation of switching onto microtubules in particular, it affected neither the speed nor the processivity of the motor on actin filaments (13). The latter suggests that the motor activity per se is not subject to phosphorylation-dependent regulation. Notably, this result was predicted by previous tracking of single melanosomes on the actin network in amphibian melanophores after removal of microtubules (14). In particular, theoretical modelling and simulations proposed that instead of regulating the motor activity, cells control the MyoVadependent switching of the organelles at actin-actin crossings to regulate the cell-wide transport (14). Upon increase of intracellular PKA activity, MyoVa was proposed to 
105 ignore the actin-actin crossings, increasing the displacement of melanosomes on the

106 actin network (14). Conversely, upon decreased PKA activity, the motor was proposed

107 to switch more frequently at actin-actin crossings, decreasing the overall displacement

108 of melanosomes (14). In the simplest scenario, however, the homodimeric MyoVa

109 motor that encounters an actin-actin crossing would statistically be expected to switch

110 with an equal probability between the same filament types. Based on our previous

111 demonstration of Mlph-regulated switching of the amphibian

112 XIRab27a/XtMlph/XIMyoVa complex at reconstituted microtubule-actin crossings (13),

113 we hypothesized that the XtMlph adaptor might also regulate the switching behavior of

114 its XIMyoVa at actin-actin crossings. To directly address this question, and to test

115 whether this presumed regulation entails any measurable impact on the overall

116 distribution of melanosomes in melanophores, here we turned to in vitro reconstitution

117 and in vivo assays, respectively. 


\section{Results and Discussion}

We previously demonstrated that the amphibian adaptor protein XtMlph from Xenopus frog interacts not only with actin filaments, but also with microtubules (13). The capability to interact with both filament types allowed XtMlph to regulate the switching probabilities of its XIMyoVa at reconstituted microtubule-actin crossings (13). In stark contrast, DrMlph-a, DrMlph-b, and DrMlph-bX2 adaptor proteins from zebrafish failed to interact with microtubules (13). All three adaptor proteins consequently failed to regulate the switching probabilities of the DrMyoVa motor at microtubule-actin intersections (13). Curiously, however, the DrMlph-bX2 acquired the capability to interact with actin filaments, as we demonstrated previously in our reconstitution assays (13). The fact that DrMlph-bX2, but not DrMlph-b, interacts with actin, is intriguing given the particularly high sequence identity between the two isoforms (95\%, Fig. S1). Identification of such isoform- and species-specific properties provides us with an opportunity to identify the structural basis of differences in Mlph function and how this was altered during vertebrate evolution.

We therefore attempted to engineer an actin-binding capability into the DrMlph-b protein based on the sequence of the DrMlph-bX2 isoform (Fig. S1). It is wellestablished that a positively charged patch in the C-terminus of Mlph is important for actin binding (Fig. S2) (15-18). Indeed, a repetitive sequence of positively charged residues is present in the C-termini of both isoforms (highlighted in red in Fig. 2A), yet, only DrMlph-bX2 interacts with actin, and not the DrMlph-b isoform (Fig. 2B, left vs. middle panel). In contrast to DrMlph-b, the positively charged patch in DrMlph-bX2 is flanked with two prolines (highlighted in bold in Fig. 2A). We reasoned that these 'rigid' prolines might 'loop out' the positively charged patch and expose it for efficient actin binding. To test this hypothesis, we simply pasted this proline patch from DrMlph-bX2 into the DrMlph-b isoform (Fig. 2A). Remarkably, this replacement alone sufficed to 
144 impart a robust actin-binding capability onto the Mlph-b isoform (Fig. 2B, middle vs.

145 right panel). Our findings thus suggest that a few strategic mutations are sufficient to

146 impart filament-binding capability and thus allow a glimpse of how the Mlph protein

147 may have started to acquire filament-binding capabilities over the course of evolution.

148 However, whether the engineered actin-binding capability into the DrMlph-b protein

149 can be ascribed solely to the exposed positively charged patch needs to be further 150 studied in the future.

A

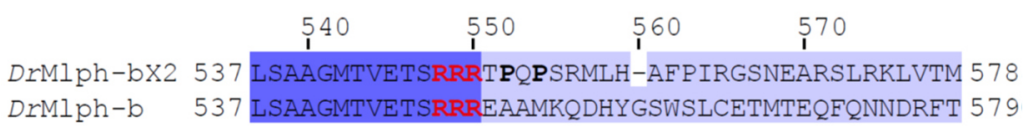

B

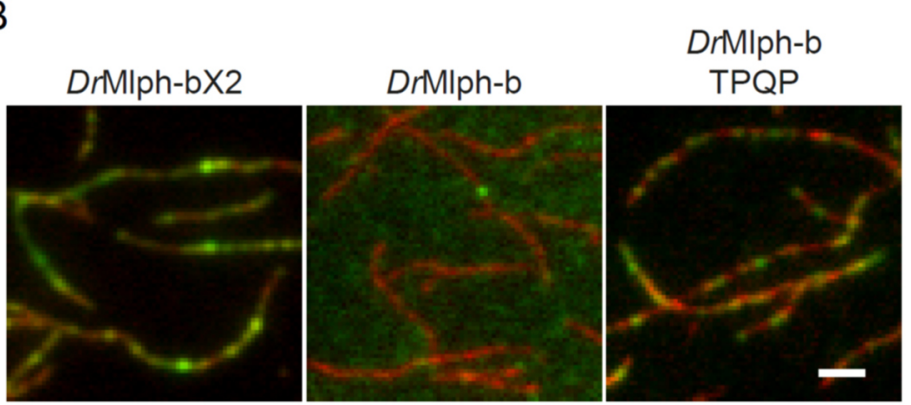

152

153

154

155

156

157

158

159

160

161

162

Fig. 2: Engineering actin-binding capability into the DrMlph-b adaptor protein. (A) Sequence alignment of DrMlph-bX2 and DrMlph-b from zebrafish, respectively. The proposed actin-binding patch is highlighted in red in the sequences. (B) Decoration of surface-attached actin filaments (red) with fluorescently labeled wild-type and mutant DrRab27a/DrMlph complexes from zebrafish (green). Left panel shows the DrMlph-bX2 adaptor protein that is capable of interacting with actin filaments, which is not observed with DrMlph-b (middle panel). (Right panel) Introducing the prolines (highlighted in bold in (A)) into the DrMlph-b protein (DrMlph-b_EAA551-553TPQP) is sufficient to prompt actin binding. Scale bar: $2 \mu \mathrm{m}$.

We next asked whether the acquired capability of DrMlph-bX2 from zebrafish to bind actin filaments regulates the switching behavior of its DrMyoVa at actin-actin crossings. To test this, we reconstituted actin-actin crossings in vitro and fluorescently labeled the DrMyoVa from zebrafish as described previously (13). When the motor arrived at an actin-actin intersection, we simply scored whether it continued on its original filament 
165

166

167

168

169

170

171

172

173

174

175

176

177

178

179

180

181

or switched onto the crossing filament (Fig. 3, left panel). As statistically expected, the uncomplexed DrMyoVa motor switched with equal probabilities at intersections that were reconstituted with the same filament type (Fig. 3A). To test our hypothesis whether the presence of the DrMlph-bX2 adaptor protein that acquired an actin-binding capability (Fig. 2B, left panel), gives rise to regulated switching at actin-actin crossings, we fluorescently labeled the DrRab27a subunit in the DrRab27a/DrMlph-bX2/DrMyoVa complex. As a control, we additionally assembled the complex with the DrMlph-b adaptor that has not yet evolved the capability to interact with actin filaments. Given that the proposed switching is regulated by the increase and decrease of intracellular PKA activity (14), we phosphorylated and dephosphorylated the DrMlph-bX2 and the DrMlph-b adaptors in the respective complexes using PKA and antarctic phosphatase as described previously (13). Consistent with our previous observations at microtubuleactin crossings, neither complexes from zebrafish displayed regulated switching behavior and both switched with equal probabilities between the actin filaments (Fig. 3B and C, Movies 1-2). Taken together, our results indicate that acquisition of actinbinding by zebrafish Mlph isoforms is insufficient to allow them to undergo PKAdependent track switching at actin-actin crossings. 


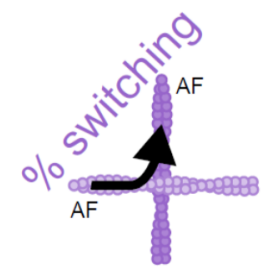

Phos.

Dephos.
A

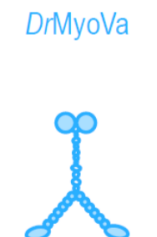

Untreated

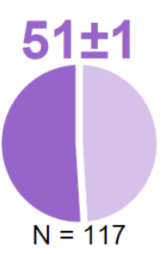

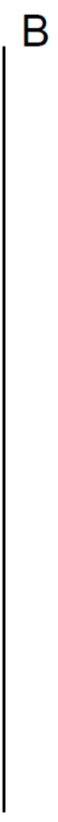
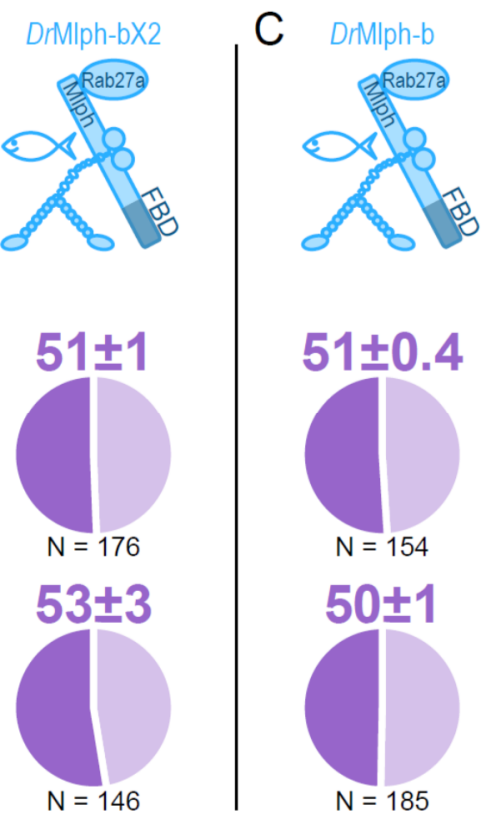

183

184

185

186

187

188

189

190

191

192

193

194

195

Fig. 3: Mlph adaptors from zebrafish fail to regulate the switching of the DrMyoVa motor at actin-actin crossings. (Top panel) Illustration of the DrMyoVa motor and respective DrRab27a/DrMlph/DrMyoVa complexes used in the study. The left column shows the switching direction and phosphorylation states (Phos. and Dephos.). Values (mean over experiments \pm $\mathrm{SD}$ ) above the pie charts represent the color-coded switching probabilities in percent. (A) Untreated DrMyoVa motor switches with equal probabilities at actin-actin crossings as statistically expected. (B and C) DrMlph-bX2 that interacts with actin filaments, as well as DrMlph-b, both fail to regulate the switching of DrMyoVa motor and the respective complexes recapitulate the switching behavior of DrMyoVa shown in (A). $N=$ number of events from 3 independent experiments. AF: actin filament.

We next turned to the amphibian model and asked whether the switching of the XIRab27a/XtMlph/XIMyoVa complex from Xenopus frog is regulated by PKA at actinactin crossings, as we demonstrated previously at microtubule-actin crossings (13). Strikingly, and in stark contrast to the zebrafish complex, about $\sim 2 / 3$ of the PKAphosphorylated Xenopus complexes ignored the actin-actin crossings and continued on the original filament (Fig. 4A, top panel, Phos.). Dephosphorylation completely reversed this behavior and $\sim 2 / 3$ of the complexes switched at the respective intersections (Fig. 4A, bottom panel, Dephos.). Further consistent with our initial hypothesis of Mlph-mediated regulation of switching behavior, removal of the C- 
terminal FBD in XtMlph abolished this regulation and the XIMyoVa motor switched with

203 equal probabilities between the actin filaments (Fig. 4B, top vs. bottom panel). The next non-trivial task will be to unmask the structural determinants of the interaction

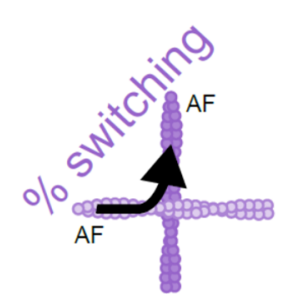
Phos.
Dephos.

\section{Dephos.}

Fig. 4: FBD of the amphibian XtMIph regulates the switching behavior of the XIMyoVa motor at actin-actin crossings. (Top panel) Illustration of the XIRab27a/XtMlph/XIMyoVa complexes used in the study. The left column shows the switching direction and phosphorylation states (Phos. and Dephos.). Values (mean over experiments \pm SD) above the pie charts represent the color-coded switching probabilities in percent. (A) (Top vs. bottom panel) Only $\sim 1 / 3$ of the PKA-phosphorylated X/Rab27a/XtMlph/XIMyoVa complexes switch at actin-actin crossings (top), and $\sim 2 / 3$ ignore the intersections and continue on their original filament. (Bottom) About $\sim 2 / 3$ of the dephosphorylated complexes switch onto the crossing filament, reversing the effect of PKA-dependent phosphorylation. (B) (Top vs. bottom panel) 
222 here directly demonstrated in in vitro reconstitution experiments (Fig. 4), affects the 223 regulated redistribution of melanosomes on the actin network. To this end, we tested 224 the effect of PKA-elevating and -lowering agents (MSH and melatonin) on melanosome distribution in microtubule-depleted Xenopus melanophores. We first confirmed the results of previous work $(11,19-26)$ showing that $\mathrm{MSH}$ and melatonin cause melanosome dispersion and clustering, respectively (Fig. 5A and B, row 1 vs. 2), and that clustering requires intact microtubules as (Fig. 5A and $B$, compare row 1 vs. 3). This indicates that melanosome dispersion seen in cells in row 3 reflects MyoVa/actindependent melanosome transport. Consistent with this notion, disruption of the actin network by cytochalasin-D treatment suppressed this dispersion and melanosomes remained clustered (Fig. 5A and $B$, row 3 vs. 4).

233 The MyoVa-mediated dispersal seen in row 3 results from the decreased PKA and 234 increased phosphatase activities, respectively. According to our experimental findings (Fig. 4) and previous simulations (14), this cell state corresponds to the dephosphorylated Rab27a/Mlph/MyoVa complex with increased switching probability 237 at actin-actin crossings (Fig. 4A, bottom panel, Dephos.). As such, the degree of dispersion should be decreased due to the frequent switching of the MyoVa motor (14). If so, solely increasing the PKA activity, thus decreasing the MyoVa switching (Fig. 4A, top panel, Phos.), is expected to further increase the degree of dispersal seen in row 3. Remarkably, MSH-dependent increase of PKA activity significantly enhanced 242 melanosome dispersion (Fig. 5A and B, row 3 vs. 5), indicating that reduced switching on the actin network enhances MyoVa/actin-driven melanosomes dispersion. Based on our reconstitution studies and consistent with previous predictions, we conclude that observed differences with respect to the displacement of melanosomes result from 246 the Mlph-regulated switching of MyoVa at actin-actin crossings, and not from the 247 regulation of the motor's activity. 
A

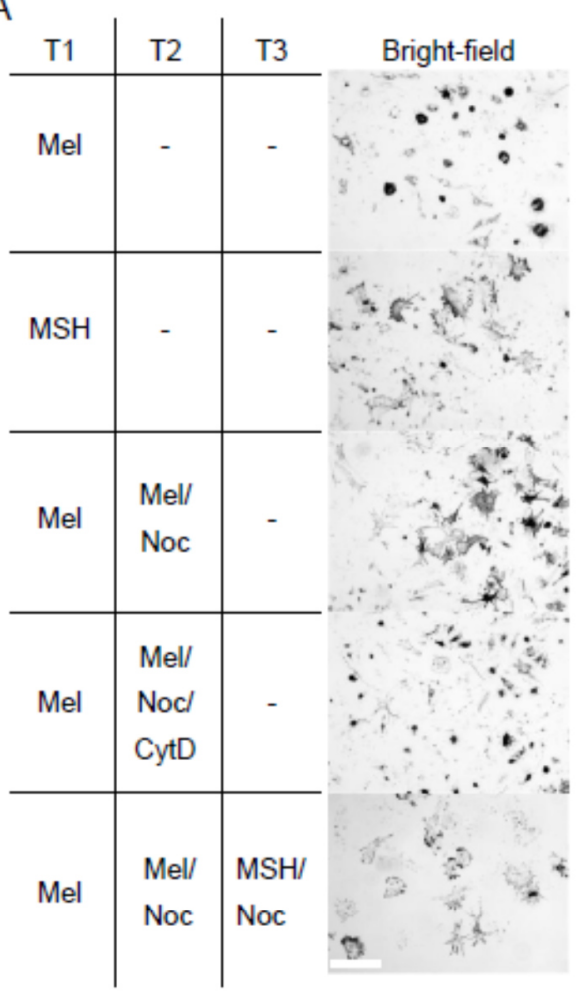

C

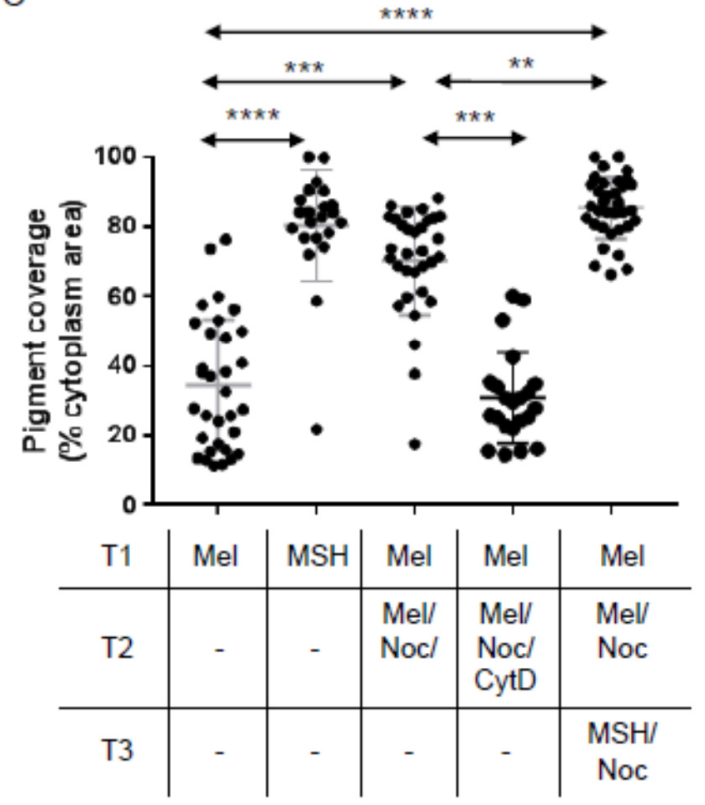

B

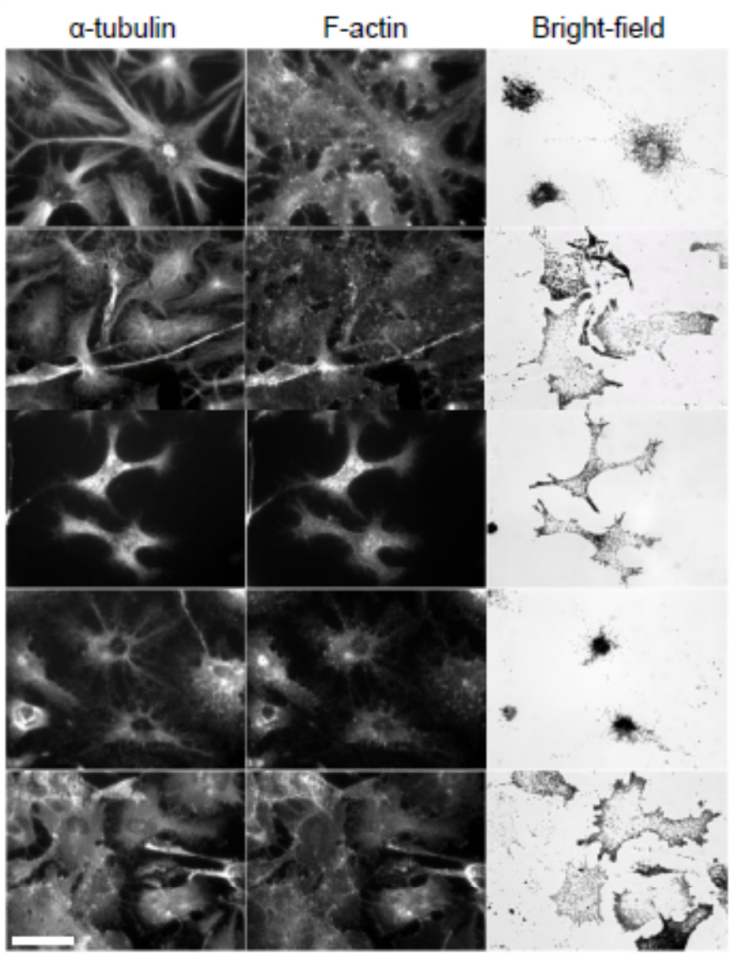

D

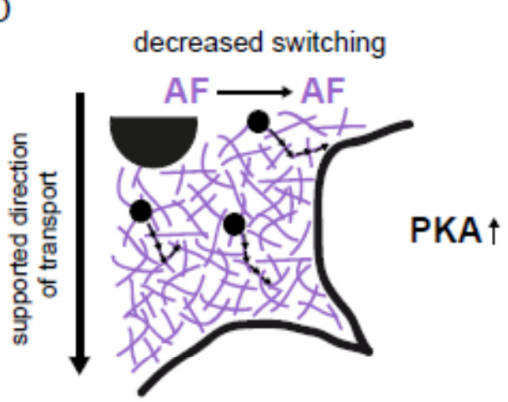

increased switching

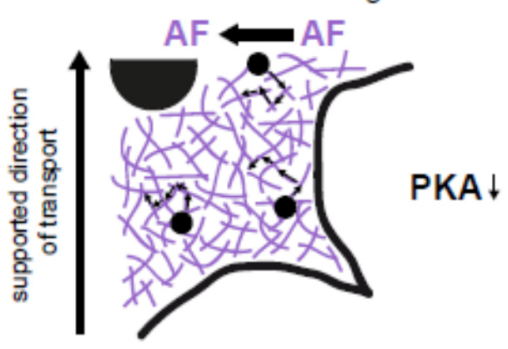

249 Fig. 5: MSH significantly enhances melanosome dispersion in microtubule-depleted

250 Xenopus melanophores in vivo. Melanophores were plated onto glass cover-slips, serum-

251 starved overnight, incubated with the indicated combination of melatonin/MSH, and

252 nocodazole/cytochalasin D, respectively, fixed after the final incubation and prepared for

253 immunofluorescence microscopy (see Materials and Methods for details). Each incubation was

254 for 1 hour at $27^{\circ} \mathrm{C}$ or $4{ }^{\circ} \mathrm{C}$ for conditions including nocodazole. (A) Left panel shows the regime

255 of incubations and the right panel shows the low magnification bright-field images of distribution of melanosomes in populations of cells in each condition, respectively. (B) High magnification 
bright-field and fluorescence images showing the distribution of melanosomes, microtubules and actin filaments in cells. (C) A scatter-plot showing the distribution of melanosomes in populations of melanophores. $\mathrm{N}=32$ (melatonin and melatonin/nocodazole), $22(\mathrm{MSH}), 22$ (melatonin/nocodazole/cytochalasin D), and 34 ( $\mathrm{MSH} /$ nocodazole). Data was analyzed using one-way ANOVA followed by Kruskal-Wallis post-test ${ }^{* *}=p<0.01,{ }^{* * *}=p<0.001$ and ${ }^{* * *}=$ $p<0.0001$. No other population comparisons yielded significant differences. Bars, $100 \mu \mathrm{m}(\mathrm{A})$ and $40 \mu \mathrm{m}$ (B). (D) Illustration of the switching regulated displacement of melanosomes as a response to intracellular PKA activity in melanophores. (Top panel) Increase of PKA activity decreases the switching probability of MyoVa and enhances the displacement of the melanosomes for dispersion. (Bottom panel) Decrease of PKA activity (i.e. transport towards the cell center for melanosome clustering) increases the switching probability and decreases the overall displacement of the organelles and contributes to aggregation. AF: actin filament.

Together with our previous findings, we propose that the regulation of MyoVa switching at microtubule-actin and actin-actin crossings both contribute to the correct positioning of melanosomes on the cytoskeleton that we now collectively trace back to the FBD of the Mlph adaptor protein (Fig. 5D). This FBD-mediated control of switching at cytoskeletal crossings observed with the amphibian XIRab27a/XtMlph/XIMyoVa complex (Fig. 4) (13) is, however, completely absent in the zebrafish complex (Fig. 3). The lack of response of the zebrafish DrRab27a/DrMlph/DrMyoVa complex to PKA also at microtubule-actin crossings (13) together suggests that the MyoVa/actin system is not yet under the control of the PKA-dependent regulation in the fish model. Previous in vivo experiments in fact delivered first indications that it is the microtubule, and not the actin network system, that is subject to regulation in fish melanophores (27). 280 Consistently, and in stark contrast to amphibians, the microtubule-based transport system alone is sufficient for hyperdispersion of melanosomes (Fig. 1C, left panel), and as such, for the voluntary color change in the fish model. Our dissection of the amphibian X/Rab27a/XtMlph/XIMyoVa complex, on the other hand, suggests that the evolution of the Mlph adaptor protein, its C-terminal FBD in particular, eventually 
specifically controls the switching behavior of the MyoVa motor not only at microtubule287 actin (13) but also at actin-actin crossings that we now reconstituted in our functional assays (Fig. 4). Importantly, both types of regulated switching on the cytoskeleton 289 support the correct overall direction of transport (Fig. 5D). Together with previous 290 findings, data presented here consistently point to a direct link between the cAMP-PKA 291 regulatory pathway and the myosin-driven transport complex. Notably, however, this 292 functional link does not 'up- and down' regulate the activity of the motor, but acts as a 293 global navigation system throughout the entire cytoskeletal lattice as a response to the 294 changes of intracellular PKA activity. 


\section{Data availability}

296 All data that support the findings of this study are available within the paper and its SI

297 Appendix.

\section{Acknowledgements}

299 We thank Vladimir I. Gelfand (Northwestern University, Chicago, U.S.A.) for his 300 generous gift of melanophore culture, and Vladimir I. Gelfand (Northwestern 301 University, Chicago, U.S.A.) and Minjong Park (University of California, San Francisco, 302 U.S.A.) for the melanophilin plasmids. We are grateful to Günther Woehlke 303 (Technische Universität München, Munich, Germany), and all members of the Ökten 304 group for fruitful discussions on the project. This work was funded by the Deutsche 305 Forschungsgemeinschaft (DFG, German Research Foundation) - SFB-863 - Project 306 ID 111166240. Z.Ö. acknowledges a starting grant from the European Research 307 Council (GA no. 335623). A.N.H. acknowledges support from MRC New Investigator 308 Award G1100063 and Wellcome Trust Institutional Strategic Support Fund award $309204843 / Z / 16 / Z$.

\section{Author Contributions}

311 A.O., P.S., D.Z., A.N.H. and Z.Ö. planned the experiments. A.O., P.S., D.Z., E.B., and 312 A.N.H. performed experiments. A.O., P.S., D.Z. and A.N.H. analyzed data. A.O., 313 A.N.H., and Z.Ö. wrote the manuscript. 
Materials and Methods

\section{Reagents}

All reagents were the highest purity available and were obtained from Sigma-Aldrich (Munich, Germany) or Carl Roth (Karlsruhe, Germany), unless mentioned otherwise.

\section{DNA constructs}

All constructs were cloned into the vector pFastBacl for subsequent expression in the baculovirus system (Life Technologies, Darmstadt, Germany). A number of constructs were codon-optimized for expression in insect cells and synthesized commercially (GenScript, Piscataway, U.S.A.). DrRab27a, DrMlph-b, DrMlph-bX2, DrMyoVa, XIRab27a, XtMlph, XtMlph $\triangle F B D$, and XIMyoVa were described earlier $(13,28)$. DrMlph-b-TPQP was synthesized with residues 551 to 554 changed to TPQP.

Protein expression, purification, fluorescent labeling, and dephosphorylation/phosphorylation and reconstitution of tripartite complexes

326 All procedures were performed as described in $(13,28)$.

Sequence alignment

MmMlph (NP_443748.2), HsMlph (NP_077006.1), FdMlph (XP_010624436.1), C/Mlph (NP_001096689.2), OaMlph (NP_001139743.1), XtMlph (NP_001120194.1), DrMlph-a (XP_005168769.1), DrMlph-b (XP_021334596.1), and DrMlph-bX2 (XP_021334597.1) were aligned using Jalview 2.10 .5 software (29) and the web service T-Coffee (30) using default parameters except gap penalty (set to 0 ).

\section{Reconstitution assay with tripartite complexes on microtubules and actin filaments}

Biotinylated Atto488-labeled microtubules and biotinylated Atto565-labeled actin filaments were attached to the surface of a flow chamber. Alexa Fluor 647-labeled dephosphorylated or phosphorylated Rab27a/Mlph/MyoVa complexes were flowed into these chambers and reconstitution assays were carried out with $4 \mathrm{mM}$ ATP as described previously (28). Movies were acquired at room temperature with the microscope setup described earlier (28). To analyze the switching behavior of the MyoVa transport complexes on actin networks, only complexes in close proximity to an actin-actin crossing were taken into account. The number of complexes that switched filament at a crossing or passed the crossing without changing filament was manually counted. Complexes that stopped their movement on a crossing were not considered as events. The switching probabilities (i.e. number of complexes that switched at actin-actin crossings divided by total number of complexes that went across crossings in \%) were determined for each complex (Dephos. And Phos.). We obtained individual switching probabilities from three independent measurements. Given switching probabilities were calculated as mean values of these individually determined switching probabilities and standard deviations were calculated using $\sigma=\sqrt{\frac{\sum_{i=1}^{n}\left(x_{i}-\mu\right)^{2}}{(n-1)}}$.

\section{Melanosome distribution assay}

For studies of melanosome distribution, melanophores were plated at a density of $1 \times 10^{4} \mathrm{cells} / \mathrm{ml}$ in growth medium into wells of a 24-well cell culture plate that were pre-loaded with uncoated $13 \mathrm{~mm}$ glass coverslips. After 24 hours, the medium was replaced with serum-free medium. Sixteen hours later this was replaced with serum-free medium supplemented with melatonin $(100 \mathrm{nM})$ or $\mathrm{MSH}(10 \mathrm{nM})$ in the presence or absence of nocodazole $(10 \mu \mathrm{M})$ or cytochalasin $D(5 \mu \mathrm{g} / \mathrm{ml})$, respectively. All incubations were for 1 hour at $27^{\circ} \mathrm{C}$ or $4^{\circ} \mathrm{C}$ for nocodazole conditions, respectively. Cells were then fixed and processed for immunofluorescence as described previously $(31,32)$. Microtubules were detected using mouse-anti-tubulin (DM1a; Calbiochem cp06) followed by Alexa488 conjugated goat anti-mouse secondary antibodies (Invitrogen A-11001) diluted at 1:100 and 1:500, respectively. F-actin was detected as previously described using Texas-red-conjugated phalloidin. Images showing melanosome, microtubule and F-actin distribution in melanophores were captured using an Axiovert S-100 microscope as described previously (33). Analysis of intracellular melanosome distribution was performed as described previously (16). Briefly, the total and melanosome filled area for individual cells were determined manually using ImageJ and pigment coverage was determined by calculating the percentage of total cell area occupied by melanosomes. 


\section{References}

1. A. N. Hume, M. C. Seabra, Melanosomes on the move: a model to understand organelle dynamics. Biochem Soc Trans 39, 1191-1196 (2011).

2. A. A. Nascimento, J. T. Roland, V. I. Gelfand, Pigment cells: a model for the study of organelle transport. Annu Rev Cell Dev Biol 19, 469-491 (2003).

3. H. Nilsson Skold, S. Aspengren, M. Wallin, Rapid color change in fish and amphibians - function, regulation, and emerging applications. Pigment Cell Melanoma Res 26, 2938 (2013).

4. V. I. Rodionov, F. K. Gyoeva, V. I. Gelfand, Kinesin is responsible for centrifugal movement of pigment granules in melanophores. Proc Natl Acad Sci U S A 88, 49564960 (1991).

5. S. L. Rogers, V. I. Gelfand, Myosin cooperates with microtubule motors during organelle transport in melanophores. Curr Biol 8, 161-164 (1998).

6. S. L. Rogers, I. S. Tint, P. C. Fanapour, V. I. Gelfand, Regulated bidirectional motility of melanophore pigment granules along microtubules in vitro. Proc Natl Acad Sci US A 94, 3720-3725 (1997).

7. M. C. Tuma, A. Zill, N. Le Bot, I. Vernos, V. Gelfand, Heterotrimeric kinesin II is the microtubule motor protein responsible for pigment dispersion in Xenopus melanophores. J Cell Biol 143, 1547-1558 (1998).

8. A. Daniolos, A. B. Lerner, M. R. Lerner, Action of light on frog pigment cells in culture. Pigment Cell Res 3, 38-43 (1990).

9. T. L. Eriksson et al., Panax ginseng induces anterograde transport of pigment organelles in Xenopus melanophores. J Ethnopharmacol 119, 17-23 (2008).

10. M. C. Isoldi, I. Provencio, A. M. Castrucci, Light modulates the melanophore response to alpha-MSH in Xenopus laevis: an analysis of the signal transduction crosstalk mechanisms involved. Gen Comp Endocrinol 165, 104-110 (2010).

11. A. R. Reilein, I. S. Tint, N. I. Peunova, G. N. Enikolopov, V. I. Gelfand, Regulation of organelle movement in melanophores by protein kinase $A(P K A)$, protein kinase $C$ (PKC), and protein phosphatase 2A (PP2A). J Cell Biol 142, 803-813 (1998).

12. V. I. Rodionov, A. J. Hope, T. M. Svitkina, G. G. Borisy, Functional coordination of microtubule-based and actin-based motility in melanophores. Curr Biol 8, 165-168 (1998).

13. A. Oberhofer et al., Molecular underpinnings of cytoskeletal cross-talk. Proc Natl Acad Sci U S A 117, 3944-3952 (2020).

14. J. Snider et al., Intracellular actin-based transport: How far you go depends on how often you switch. P Natl Acad Sci USA 101, 13204-13209 (2004).

15. M. Fukuda, T. S. Kuroda, Slac2-c (synaptotagmin-like protein homologue lacking C2 domains-c), a novel linker protein that interacts with Rab27, myosin Va/VIla, and actin. J Biol Chem 277, 43096-43103 (2002).

16. A. N. Hume, A. K. Tarafder, J. S. Ramalho, E. V. Sviderskaya, M. C. Seabra, A coiledcoil domain of melanophilin is essential for Myosin Va recruitment and melanosome transport in melanocytes. Mol Biol Cell 17, 4720-4735 (2006).

17. T. S. Kuroda, H. Ariga, M. Fukuda, The actin-binding domain of Slac2-a/melanophilin is required for melanosome distribution in melanocytes. Mol Cell Biol 23, 5245-5255 (2003). 
18. M. Sckolnick, E. B. Krementsova, D. M. Warshaw, K. M. Trybus, More than just a cargo adapter, melanophilin prolongs and slows processive runs of myosin $\mathrm{Va}$. J Biol Chem 288, 29313-29322 (2013).

19. K. Abe et al., Role of cyclic AMP in mediating the effects of MSH, norepinephrine, and melatonin on frog skin color. Endocrinology 85, 674-682 (1969).

20. P. N. de Graan, A. N. Eberle, Irreversible stimulation of Xenopus melanophores by photoaffinity labelling with p-azidophenylalanine13-alpha-melanotropin. FEBS Lett 116, 111-115 (1980).

21. B. Magun, Two actions of cyclic AMP on melanosome movement in frog skin. Dissection by cytochalasin B. J Cell Biol 57, 845-858 (1973).

22. M. N. Potenza, M. R. Lerner, A rapid quantitative bioassay for evaluating the effects of ligands upon receptors that modulate cAMP levels in a melanophore cell line. Pigment Cell Res 5, 372-378 (1992).

23. D. Sugden, S. J. Rowe, Protein kinase C activation antagonizes melatonin-induced pigment aggregation in Xenopus laevis melanophores. J Cell Biol 119, 1515-1521 (1992).

24. T. Ebisawa, S. Karne, M. R. Lerner, S. M. Reppert, Expression cloning of a high-affinity melatonin receptor from Xenopus dermal melanophores. Proc Natl Acad Sci U S A 91, 6133-6137 (1994).

25. A. B. Lerner, J. D. Case, Y. Takahashi, Isolation of melatonin and 5-methoxyindole-3acetic acid from bovine pineal glands. J Biol Chem 235, 1992-1997 (1960).

26. B. H. White, R. D. Sekura, M. D. Rollag, Pertussis toxin blocks melatonin-induced pigment aggregation in Xenopus dermal melanophores. J Comp Physiol B 157, 153159 (1987).

27. B. M. Slepchenko, I. Semenova, I. Zaliapin, V. Rodionov, Switching of membrane organelles between cytoskeletal transport systems is determined by regulation of the microtubule-based transport. J Cell Biol 179, 635-641 (2007).

28. A. Oberhofer et al., Myosin Va's adaptor protein melanophilin enforces track selection on the microtubule and actin networks in vitro. P Natl Acad Sci USA 114, E4714-E4723 (2017).

29. A. M. Waterhouse, J. B. Procter, D. M. Martin, M. Clamp, G. J. Barton, Jalview Version 2--a multiple sequence alignment editor and analysis workbench. Bioinformatics 25, 1189-1191 (2009).

30. C. Notredame, D. G. Higgins, J. Heringa, T-Coffee: A novel method for fast and accurate multiple sequence alignment. J Mol Biol 302, 205-217 (2000).

31. A. N. Hume, D. S. Ushakov, A. K. Tarafder, M. A. Ferenczi, M. C. Seabra, Rab27a and MyoVa are the primary Mlph interactors regulating melanosome transport in melanocytes. J Cell Sci 120, 3111-3122 (2007).

32. R. D. Evans et al., Myosin-Va and dynamic actin oppose microtubules to drive longrange organelle transport. Curr Biol 24, 1743-1750 (2014).

33. C. L. Robinson, R. D. Evans, D. A. Briggs, J. S. Ramalho, A. N. Hume, Inefficient recruitment of kinesin-1 to melanosomes precludes it from facilitating their transport. $J$ Cell Sci 130, 2056-2065 (2017). 